Projets

de paysage

\section{Projets de paysage}

Revue scientifique sur la conception et l'aménagement de l'espace

13 | 2015

Biodiversité et paysage

\title{
La trame arborée : un élément pertinent pour articuler paysage et biodiversité dans la politique de la trame verte et bleue aux échelles infrarégionales?
}

The Green Corridor : A Relevant Element for Combining Landscape and Biodiversity in the Green and Blue Infrastructure Policy at the Sub-Regional Level

Éric Maire, Philippe Béringuier, Gérard Briane, Bertrand Desailly et Sylvie Guillerme

\section{OpenEdition}

Édition électronique

URL : http://journals.openedition.org/paysage/9323

DOI : $10.4000 /$ paysage. 9323

ISSN : 1969-6124

Éditeur :

École nationale supérieure du paysage de Versailles-Marseille, Institut national des sciences appliquées Centre Val de Loire - École de la nature et du paysage, École nationale supérieure d'architecture et de paysage de Bordeaux, École nationale supérieure d'architecture et de paysage de Lille, Agrocampus Angers

\section{Référence électronique}

Éric Maire, Philippe Béringuier, Gérard Briane, Bertrand Desailly et Sylvie Guillerme, « La trame arborée : un élément pertinent pour articuler paysage et biodiversité dans la politique de la trame verte et bleue aux échelles infrarégionales ? », Projets de paysage [En ligne], 13 | 2015, mis en ligne le 31 décembre 2015, consulté le 04 septembre 2020. URL : http://journals.openedition.org/paysage/9323 DOI : https://doi.org/10.4000/paysage.9323

Ce document a été généré automatiquement le 4 septembre 2020

Projets de paysage 


\section{La trame arborée : un élément} pertinent pour articuler paysage et biodiversité dans la politique de la trame verte et bleue aux échelles infrarégionales?

The Green Corridor : A Relevant Element for Combining Landscape and Biodiversity in the Green and Blue Infrastructure Policy at the Sub-Regional Level

Éric Maire, Philippe Béringuier, Gérard Briane, Bertrand Desailly et Sylvie Guillerme

1 La trame verte et bleue (TVB) issue du Grenelle II de l'environnement (2010) est couramment présentée comme une politique environnementale innovante. Elle s'impose, à travers les schémas régionaux de cohérence "écologique (SRCE), aux documents d'aménagement et d'urbanisme : schémas de cohérence territoriale (Scot) et plans locaux d'urbanisme communal (PLU) et intercommunal (PLUI). Cette politique publique, originale au niveau européen, vise à préserver ou à restaurer les continuités écologiques terrestres et aquatiques, afin de connecter les différents réservoirs de biodiversité (Clergeau et Désiré, 1999 ; Clergeau, 2007 ; Bonnin, 2008 ; Clergeau et Blanc, 2013). Toutefois, les connaissances scientifiques sur l'efficacité des corridors écologiques sont encore insuffisantes (Vanpeene-Bruhier, 2014). De récents travaux ont, en outre, montré que cette politique pouvait parfois être mal perçue par les acteurs locaux (Cormier, 2011). Aussi, la notion de trame verte est fréquemment comprise de manière différente selon les acteurs. 
2 Pour les besoins de l'article et afin de mieux cibler les enjeux spécifiques associés à la trame arborée, il convient de bien séparer les différents constituants de la trame verte et bleue :

- la trame arborée (TA) correspond à la composante ligneuse, arborée et arbustive, c'est-àdire les haies, les arbres hors forêt depuis les arbres de haut jet jusqu'aux arbustes, les bosquets et les ensembles forestiers ;

- la trame verte (TV) comprend la trame arborée (TA), les prairies et pelouses, les cultures pérennes, les bandes enherbées, les jardins, voire certaines cultures temporaires ;

- la trame bleue (TB) est composée du réseau hydrographique, des étendues d'eau (lacs, étangs) et des zones humides (tourbières, marais, etc.) ;

- la trame verte et bleue (TVB) procède de la définition du Grenelle II de l'environnement ${ }^{1}$, et associe TV et TB.

3 La trame arborée, en tant que structure paysagère, est donc une composante essentielle des corridors écologiques définis par la politique TVB. Elle constitue, de par sa complexité structurelle et physionomique (arbres, arbustes, strates herbacées associées), un élément pertinent d'analyse de la biodiversité au sein d'une structure paysagère complexe. Elle est essentielle au maintien de la biodiversité dite « ordinaire ». Françoise Burel et Jacques Baudry (1989) ont ainsi montré que les haies servaient de couloirs pour les coléoptères carabiques forestiers. Alain Butet et al. (2006) ont observé le même phénomène à propos des petits mammifères. Ces études soulignent le rôle clé de la trame arborée pour la circulation des espèces.

4 L'identification aux échelles les plus fines des composantes de la TVB, en particulier celles relevant de la trame arborée, se heurte cependant à un certain nombre de verrous méthodologiques (Maire et al., 2012). Les cartographies et préconisations de grands corridors écologiques à une échelle atteignant le 1/50 000 dans le meilleur des cas ont certes leur utilité, comme outils de communication et de planification à l'échelle régionale, mais elles ne reflètent pas réellement les enjeux locaux de biodiversité, comme le montre le guide méthodologique proposé par la direction régionale de l'Environnement, de l'Aménagement et du Logement (Dreal) MidiPyrénées $^{2}$. Or, la porosité des politiques publiques de la TVB vers les plans locaux d'urbanisme (PLU) se fait selon le principe de la subsidiarité. Les acteurs locaux, malgré les documents de connaissance et d'aide à la décision, ne disposent pas actuellement des moyens techniques qui leur permettraient de réaliser des cartographies plus précises, qui pourtant s'avéreraient fort utiles pour la mise en œuvre de la TVB sur le terrain.

5 À travers un exemple pris dans le piémont pyrénéen ariégeois et haut-garonnais (vallée $\mathrm{du}$ Salat), zone en déprise agricole à l'interface du parc naturel régional des Pyrénées ariégeoises et de secteurs ne faisant pas l'objet de politiques de protection environnementale spécifiques, nous montrerons dans un premier temps qu'il existe des méthodes relativement simples pour réaliser des cartographies fines de la trame arborée, soit en utilisant la photo-interprétation, soit en ayant recours à des images des satellites Pléiades ${ }^{3}$. Cette approche panoptique des structures paysagères pourrait ainsi préfigurer une future "TVB haute résolution» à l'échelle nationale. Une telle cartographie présenterait aussi l'avantage d'être plus «parlante » pour les acteurs locaux, car a priori davantage en adéquation avec leur territoire de proximité.

6 Dans un second temps, l'analyse s'appuiera sur les résultats d'un atelier participatif réalisé au printemps 2014, au cours duquel des acteurs locaux ont été invités, d'une 
part, à réagir aux cartes « expertes » que nous leur avons présentées, d'autre part, à réaliser à leur tour des représentations cartographiques. Force est de constater que le niveau de précision des cartes à haute résolution suscite quelques inquiétudes parmi les acteurs locaux, ce qui peut aller à l'encontre de l'un des objectifs poursuivis : faciliter la mise en place de la TVB au niveau local. Nous montrerons ainsi combien la carte est susceptible de révéler des enjeux environnementaux, paysagers et territoriaux différents selon les acteurs concernés.

Les résultats présentés ici sont issus du projet de recherche Interface, réalisé dans le cadre du programme "Paysage et développement durable» (PDD2) du ministère de l'Écologie, du Développement durable et de l'Énergie. Ce projet portait sur le rôle de l'arbre hors forêt (AHF) comme révélateur de multiples enjeux territoriaux liés à la biodiversité (Guillerme, 2014).

\section{Une enquête dans le piémont pyrénéen}

\section{La basse vallée du Salat : un espace de transitions}

Le terrain d'étude, la basse vallée du Salat, apparaitt à plusieurs titres comme un espace de transitions. Il appartient en premier lieu au piémont pyrénéen, entre la haute montagne au sud de Saint-Girons et la plaine de la Garonne au nord. La rivière Salat sinue au sein d'un couloir à fond plat, large de deux à trois kilomètres, encadré par les collines des Petites Pyrénées avoisinant 500 mètres d'altitude.

Figure 1. Carte de localisation

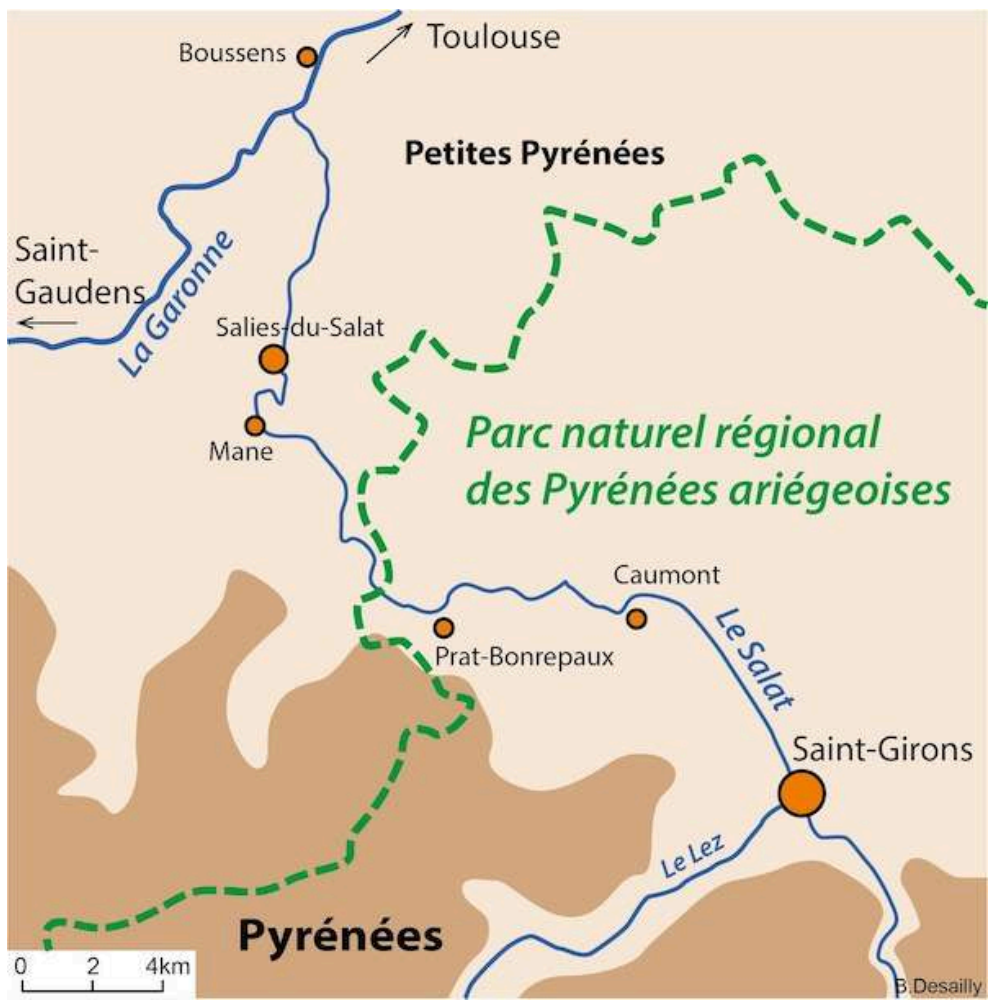

Le contraste n'est pas seulement topographique. Le fond de la vallée, occupé principalement par des champs et des prairies, localement par quelques peupleraies, 
est jalonné de villages peuplés chacun de mille à deux mille habitants tout au plus : Prat-Bonrepaux, Mane, Salies-du-Salat. La proximité relative de Toulouse (une heure de trajet par l'autoroute A64) permet également les migrations pendulaires. Les constructions récentes restent cependant éparses et la démographie peu dynamique : la population stagne ou est en léger repli. Le constat vaut aussi pour les villages beaucoup plus modestes des Petites Pyrénées, d'accès malcommode, qui ne comptent souvent que quelques dizaines d'habitants. Bois de chênes et friches sont très présents sur les collines, conquérants, réduisant peu à peu les clairières : image classique d'un contexte de déprise agricole.

La transition est aussi temporelle et économique. La basse vallée du Salat a vécu un siècle et demi durant au rythme de l'industrie papetière, qui a occupé une position majeure au niveau national et même international grâce à une spécialité : le papier à cigarettes (Minovez, 2014). Des usines donc, concentrées autour de Saint-Girons et de Mazères-sur-Salat, mais point de cités ouvrières : la main-d'œuvre était constituée d'ouvriers logeant dans les villages alentour, ayant conservé une activité agricole secondaire. De cette dernière subsistent encore quelques témoins, notamment quelques vergers et petites parcelles de vigne, dont le manque d'entretien laisse augurer la disparition prochaine. L'activité papetière a connu un long déclin après la Seconde Guerre mondiale, et le tournant $\mathrm{du} \mathrm{xxI}^{\mathrm{e}}$ siècle, avec la fermeture de plusieurs sites majeurs, marque la fin d'un cycle économique: aux friches agricoles des coteaux répondent aujourd'hui les friches industrielles de la vallée. Si le secteur agricole, centré sur l'élevage bovin, continue d'occuper quelques actifs, l'économie résidentielle a désormais pris la place de l'industrie.

11 La marque de l'industrie dans les paysages se lit aussi dans la rivière. L'installation des papeteries a nécessité la construction d'ouvrages hydrauliques. De nombreux barrages et chaussées d'anciens moulins barrent la rivière, souvent équipés aujourd'hui pour la production électrique. Des gravières importantes ont par ailleurs été exploitées à l'aval, au voisinage de Salies-du-Salat. Le Salat est donc une rivière fortement anthropisée, dont la pollution due à l'activité papetière a été réduite et qui présente des espèces emblématiques comme la loutre d'Europe ainsi qu'une bonne conservation localement de certains habitats, tels que méandres, îles fluviales et ripisylves ${ }^{4}$. L'intérêt floristique et faunistique du Salat et de ses berges a ainsi justifié leur intégration en 2009 à un site Natura 2000, aux côtés de la Garonne et d'autres rivières pyrénéennes.

12 Considéré sous l'angle de la trame arborée, le bas Salat offre ainsi un tableau contrasté. Hors de la vallée, en présence d'une végétation ligneuse envahissante, l'enjeu est plutôt le maintien de milieux. 
Figure 2. Le bassin de Cazavet

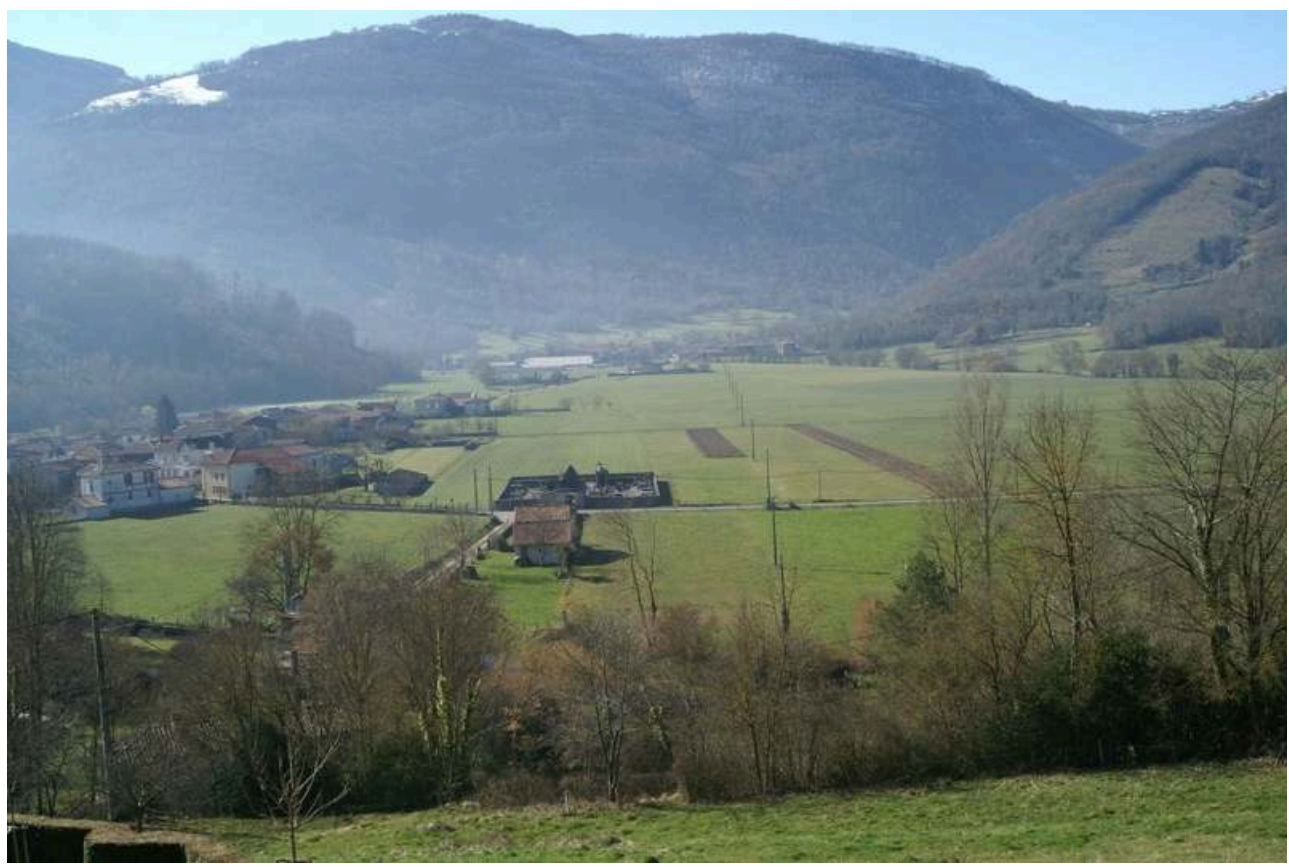

Les clairières des Petites Pyrénées ont par ailleurs conservé un aspect bocager, arbres et arbustes sont nombreux en limite de parcelles. À l'inverse, haies, bosquets et arbres isolés ont été éliminés des larges parties du fond de vallée, qui offrent aujourd'hui un paysage dégagé. C'est notamment le cas dans les petites plaines alluviales de Caumont, à l'aval de Saint-Girons et de Prat-Bonrepaux, consacrées aujourd'hui notamment à la culture du maïs irrigué. C'est alors la rivière et sa végétation rivulaire qui forment la principale continuité écologique.

Un dernier élément de contexte vaut d'être mentionné : il s'agit de la création en 2009 du parc naturel régional des Pyrénées ariégeoises. Celui-ci présente un vaste périmètre puisqu'il englobe toute la moitié ouest du département de l'Ariège, laissant à l'écart à l'est l'axe du Val d'Ariège, dominé par les villes de Foix et Pamiers. Le parc s'étend à la fois sur la haute chaîne et son piémont, et l'ensemble des communes ariégeoises du bas Salat, de Saint-Girons à La Bastide-du-Salat, en fait partie. Les communes à l'aval, situées en Haute-Garonne, ne sont pas incluses dans le parc. La création des deux départements en 1790, reprenant la limite entre les anciens pays du Couserans et du Comminges, a eu en effet pour résultat de couper en deux la vallée. Une telle situation pourrait aujourd'hui avoir des répercussions sur les actions menées dans les domaines de l'environnement, du paysage et du patrimoine. À moins que voie le jour un PNR du Comminges, couvrant le Sud du département de la Haute-Garonne, le projet, depuis longtemps dans les cartons, peine à se concrétiser, notamment en raison du contexte politique local.

\section{Méthodologie}

La méthodologie mise en œuvre fait appel aux données et cartographies scientifiques et institutionnelles, ainsi qu'à un travail mené avec des acteurs locaux. Nous avons cherché à caractériser leurs représentations lorsqu'ils s'expriment sur le paysage en relation avec la composante arborée, en mettant en place un dispositif associant la 
création de cartes à haute résolution, et la production de cartes à dire d'acteurs lors d'un atelier mené en juin 2014.

\section{Une cartographie fine de la trame arborée} verte et bleue et de sa principale composante, la trame arborée, serait un outil essentiel pour renforcer la cohérence des futures politiques de biodiversité et agroenvironnementales, nous avons cherché à cartographier ces éléments avec une précision métrique. Une première exploration des méthodes de télédétection en résolution métrique a été testée sur une image Quick Bird fournie via un travail de collaboration avec le Centre d'études spatiales de la Biosphère (Cesbio) dans le cadre du projet Orfeo (Cnes) (Maire et al., 2012). Puis le travail a été mené sur des images métriques multispectrales du satellite Pléiades sur la basse vallée du Salat. La cartographie à haute résolution consiste à utiliser des algorithmes de télédétection et des techniques de cartographie basées sur les systèmes d'information géographique (SIG). La démarche doit s'appuyer sur les images satellites les plus récentes possibles. En effet, le couvert arboré évolue parfois très rapidement et la trame ligneuse arbustive - surtout quand elle est principalement composée de haies basses - n'est que peu cartographiée sur une grande emprise spatiale. C'est une des raisons pour lesquelles la couche « végétation » de la BD-Topo de l'IGN est insuffisante pour montrer les emprises réelles du couvert arboré à l'échelle communale, voire parcellaire.

Les cartes d'occupation du sol étaient auparavant les documents privilégiés pour montrer les enjeux de biodiversité. Elles sont le plus souvent produites à la fois par télédétection et par des validations de terrain. Des données à l'échelle européenne ont été jusqu'ici largement utilisées, comme CORINE Land Cover dont la plus petite unité est de 25 ha. La connaissance de l'occupation du sol à grande échelle (OCSGE ${ }^{5}$ ) est un enjeu national, susceptible de répondre aux besoins des élus dans leurs processus de décision, afin par exemple de limiter l'artificialisation des territoires. Toutefois, la trame arborée hors forêt, incluant les haies, arbres isolés, petits bosquets, est mal cartographiée, sinon tout simplement absente dans le cas de résolutions supérieures à environ $8 \mathrm{~m} /$ pixel.

Cependant, les cartes de la trame arborée trouvent de plus en plus leur place dans la compréhension des enjeux environnementaux et, en conséquence, diverses méthodologies ont vu le jour, qui s'affinent sans cesse (Sheeren et al., 2009; Tansey et al., 2009). Outre des algorithmes de classification de plus en plus adaptés, ces méthodologies offrent à la fois un volet instrumental - l'usage des capteurs haute résolution capables de produire des images multispectrales telles les images Pleiades et un volet «multistrate » haute résolution en déployant la technique laser aéroporté (Lidar). Cette dernière technique n'a toutefois pas été utilisée ici car onéreuse et générant de très grandes quantités de données, dès lors qu'on travaille sur des terrains assez étendus.

19 Pour la cartographie de la trame arborée à partir d'images satellites, nous avons eu recours à des méthodes de classification supervisée (Richard et Xiuping, 2006) et de morphologie mathématique (Serra, 1982; Maire et al., 2012). Le travail préalable a été de créer une mosaïque à partir d'un maximum d'images couvrant le terrain d'étude. Ensuite, une classification supervisée «Parallelepiped Classification» a été appliquée. Le résultat ainsi obtenu est une cartographie à la fois des espaces boisés et forestiers, 
des haies épaisses, des ripisylves, des grands arbres isolés et des petits bosquets. Les haies d'arbustes d'épaisseur inférieure à 3 mètres sont cependant trop fines pour être détectées avec de telles images.

\section{Un atelier participatif}

Afin d'explorer les représentations spatiales des acteurs locaux lorsqu'ils s'expriment sur la trame arborée, nous avons organisé en juin 2014 un atelier participatif sur le terrain de l'étude. Cet atelier d'une journée, intitulé «Mots et lieux de la trame arborée ", a réuni 20 personnes et s'est déroulé dans la commune de Prat-Bonrepaux (Guillerme et al., 2014). L'objectif était de mettre en évidence la diversité des représentations de la trame arborée et des enjeux spatiaux de paysage et de biodiversité qui lui sont associés, dans le cadre d'une approche qualitative avec un nombre restreint de participants ayant un profil diversifié et issus d'horizons différents: agriculteurs, élus locaux, membres d'associations de protection du patrimoine ou de l'environnement, techniciens intervenant dans les domaines de la gestion des forêts ou des cours d'eau. Dans un contexte de petites communes rurales, plusieurs participants occupaient simultanément différentes fonctions (forestier et élu local par exemple).

Durant cet atelier, la carte a été utilisée à la fois comme outil d'expression et de dialogue.

Figure 3. Réalisation des cartes par les participants de l'atelier

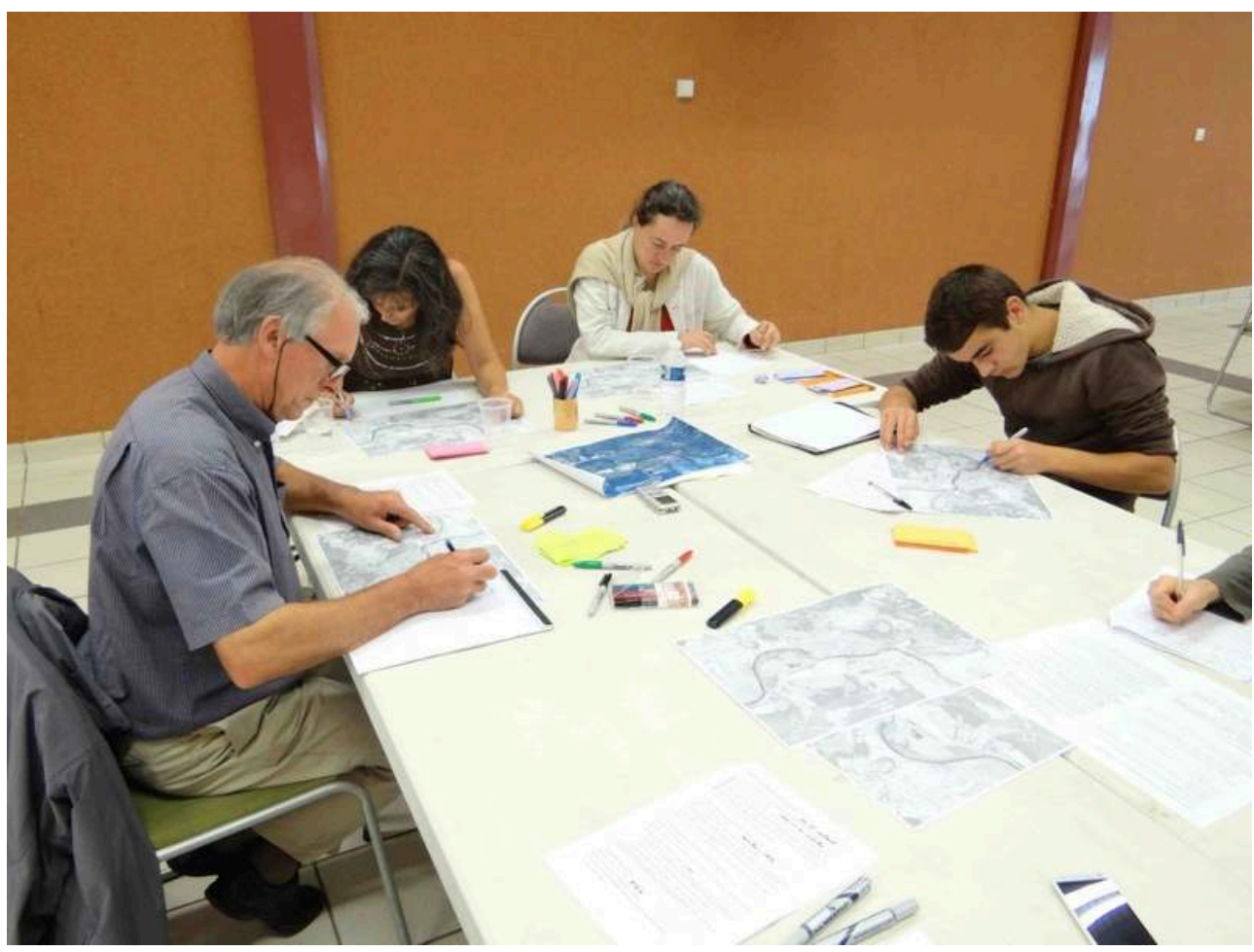


Figure 4. Les cartes mises en débat

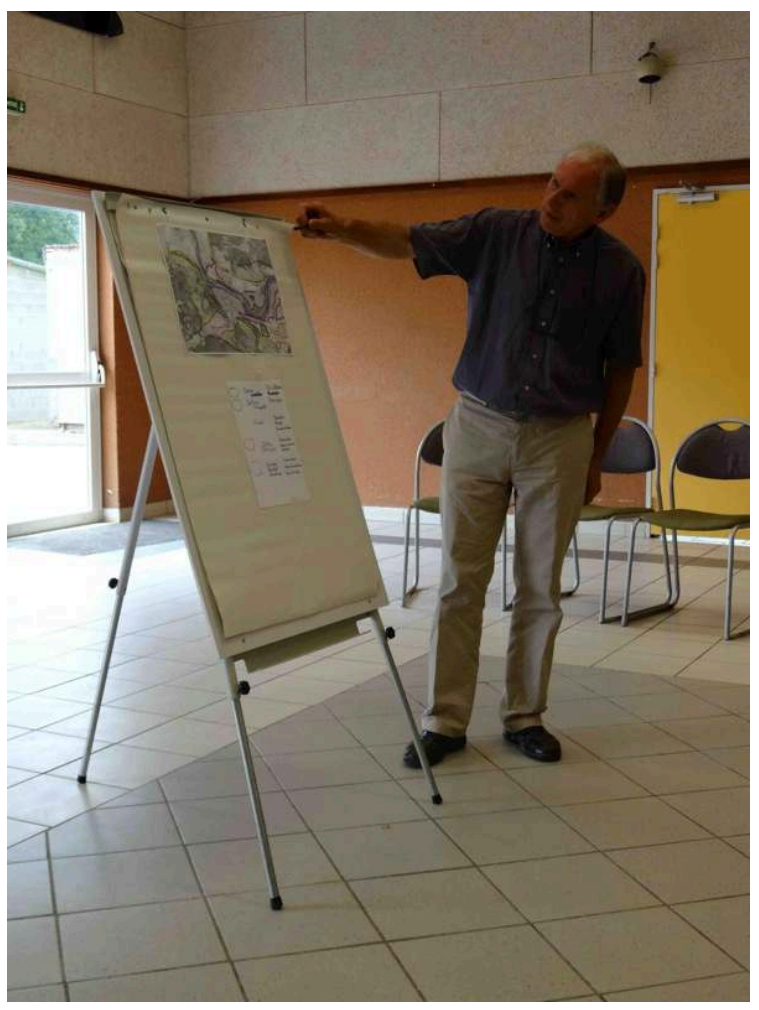

21 Les principes de travail, collecte et exploitation des données, sont inspirés de différents travaux-ateliers conduits antérieurement dans le cadre du réseau paysage MidiPyrénées et de recherches-action (Lelli et al., 2012 ; Beringuier et al., 2014). Dans un premier temps, on a demandé à chaque participant de réaliser individuellement une carte de la "trame arborée » - nous avons volontairement employé l'expression, sans en fournir dans un premier temps une définition précise - accompagnée de sa légende, sur une feuille A3 comportant en fond une photographie aérienne noir et blanc. La zone couverte, de quelques kilomètres carrés, était centrée sur le village de Prat-Bonrepaux et comportait une certaine diversité d'éléments arborés: bois, éléments de haies, ripisylves, arbres isolés... Nous avons ensuite, d'abord en sous-groupes puis tous ensemble, confronté les réalisations de chacun et les points de vue sur d'autres cartes, celles issues du SRCE et les cartes à haute résolution que nous avions produites (cf. «Une cartographie fine de la trame arborée »).

\section{Le choc des cartes}

\section{Cartographier la trame arborée à haute résolution}

22 Nous avons cartographié par classification supervisée la trame arborée sur de vastes surfaces d'environ $100 \mathrm{~km}^{2}$ en résolution moyenne de $5 \mathrm{~m} /$ pixel. Outre les noyaux villageois, ces territoires sont composés majoritairement à la fois de terres agricoles, de structures arbustives et de petits bosquets. Nous avons ensuite opéré des zooms sur les zones qui ont servi de support aux ateliers cartographiques avec les acteurs locaux. Cette seconde cartographie a été obtenue par télédétection à partir des images Pléiade de résolution $0,65 \mathrm{~m} /$ pixel. On observe, d'une part, une meilleure précision des 
contours à haute résolution et, d'autre part, la présence d'arbres isolés et d'arbres de vergers, absents de la première carte.

Figure 5. Comparaison des classifications de la trame arborée dans la basse vallée du Salat, secteur de Prat-Bonrepaux (Ariège)
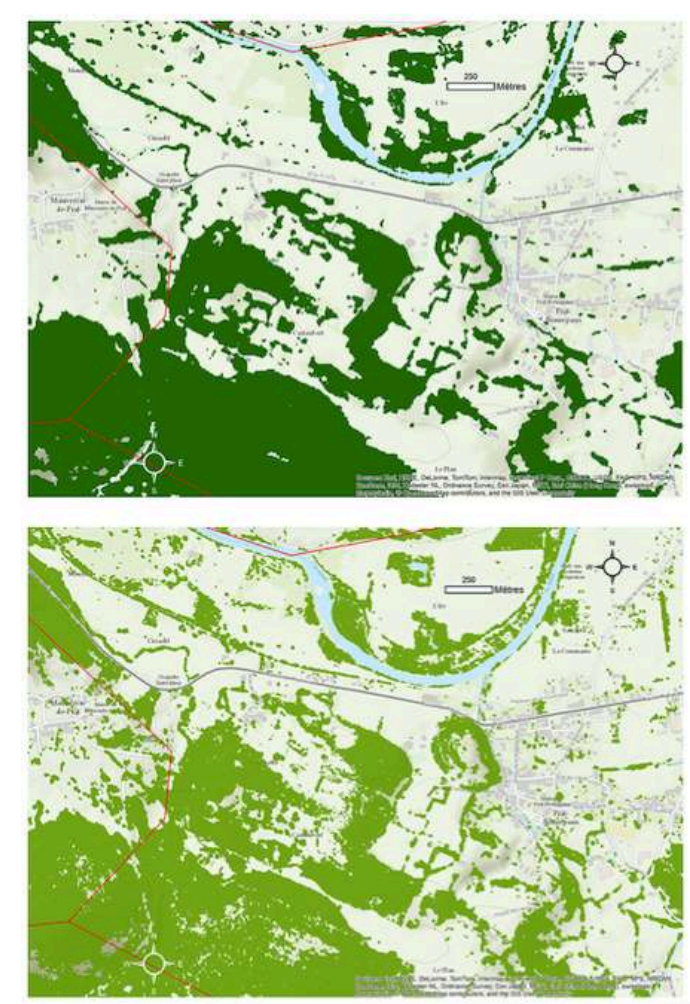

En vert foncé, la couche arborée a été obtenue à partir d'images RapidEye à 5 m/pixel (juillet 2012) ; en vert clair, la couche arborée a été obtenue à partir d'images Pleiades à 0,65 m/pixel (juin 2013).

Cette cartographie à haute résolution pourrait constituer un outil de grande précision à une échelle locale. Au-delà des PLU/PLUI ou des cartes communales, les collectivités pourraient ainsi, grâce à une meilleure connaissance spatiale, disposer d'éléments afin de définir des actions permettant de limiter l'érosion de la biodiversité, ou de proposer des plans pour la conservation, la restauration ou la création de continuités écologiques sur leur territoire. À cet égard, il est possible de superposer les points de friction, de conflits potentiels (ou risques de césure du corridor écologique) inscrits dans le SRCE de ce secteur à l'échelle la plus fine afin de mieux comprendre ce que cela implique d'un point de vue de la structuration de la trame arborée. 
Figure 6. Photographie aérienne (IGN 2013) du secteur de Prat-Bonrepaux

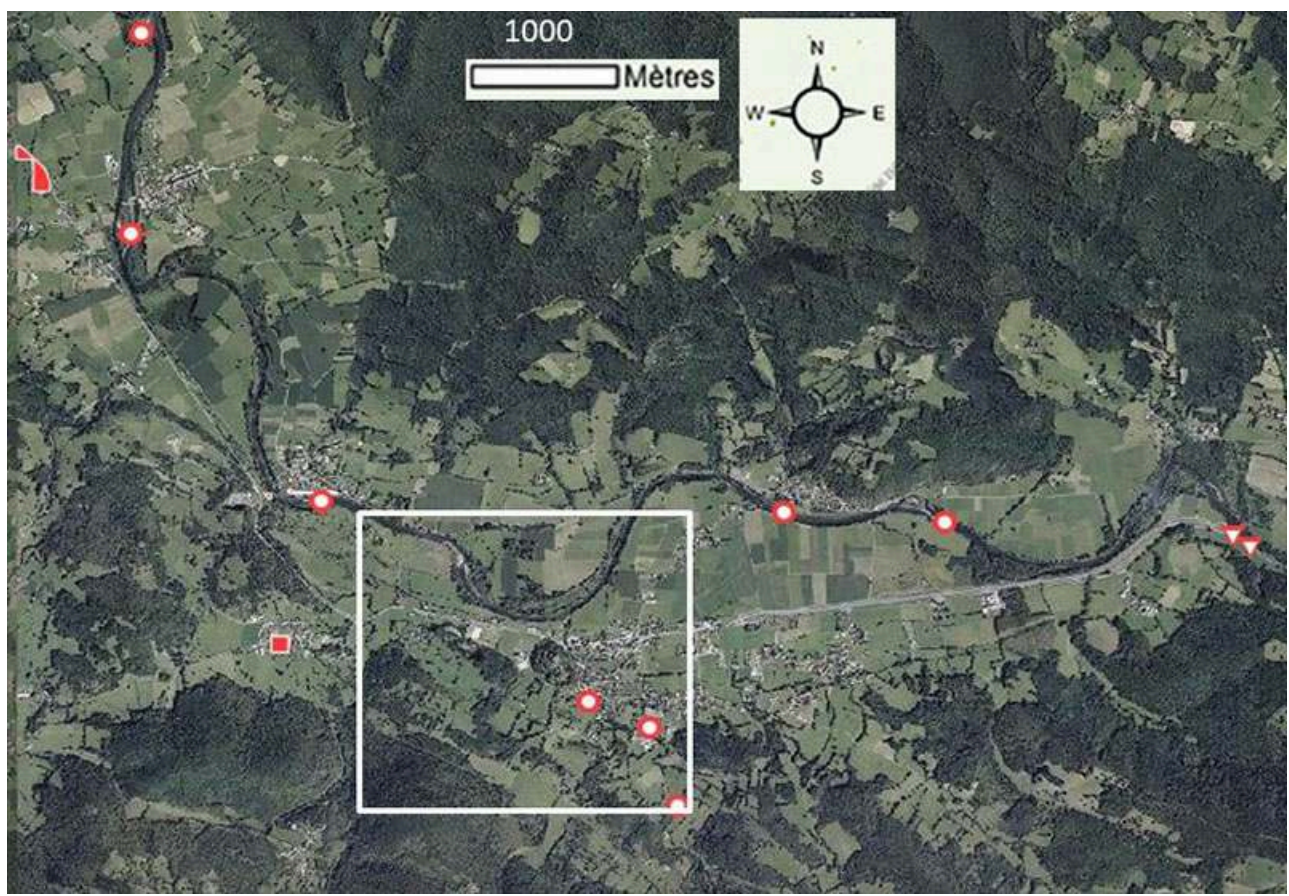

Avec (superposés en rouge) les points de conflits potentiels provenant du SRCE Midi-Pyrénées conduisant à un risque de discontinuité d'un corridor écologique. Les triangles concernent les passages de type corridor entre grand ensemble forestier de part et d'autre de la vallée (à gauche). Les ronds concernent les obstacles à l'écoulement des eaux (trame bleue). Les autres zones en rouge concernent la présence de zones urbanisées obérant la continuité d'un corridor. La partie délimitée par le rectangle blanc (commune de Prat-Bonrepaux) correspond à la fois à la zone ayant fait l'objet de la cartographie haute résolution de la trame arborée et la zone d'intérêt pour l'atelier de cartographie avec les acteurs locaux.

On constate que les zones de potentielles ruptures de corridors écologiques mises en exergue dans le SRCE se retrouvent peu nombreuses à l'échelle d'une commune et donc d'un PLU/PLUI. Il apparaît que le SRCE ne s'occupe que des connectivités générales des corridors écologiques, lesquelles ne parlent guère aux acteurs locaux en termes d'enjeux cartographiables à l'échelle d'un PLU. Ainsi, la question de l'applicabilité et de la déclinaison de la politique de la trame verte et bleue à l'échelon local, notamment la porosité des recommandations des SRCE et des Scot vers les PLU et PLUI, souffre d'une discordance d'échelles. Les SRCE sont au mieux au 1/100 000, alors que le PLU nécessite une échelle inférieure au 1/50000 en raison des obligations de représentation spatialisée des parcelles cadastrales.

Lors de l'atelier, les cartes haute résolution créées par télédétection ont été proposées aux acteurs présents afin de recueillir leur point de vue. Ces images, faisant apparaître la trame arborée, ont été perçues de façon contrastée. Elles traduisent des visions tantôt partagées, tantôt plus spécifiques selon le profil de chaque acteur.

Positivement, elles ont été unanimement perçues comme :

- un progrès dans la connaissance pour la mise en œuvre à échelle fine de mesures favorables à la biodiversité. Ce point de vue est surtout celui des acteurs et des professionnels directement concernés par la protection environnementale ;

- un outil intéressant pour une meilleure connaissance de la circulation de la faune ;

- offrant des possibilités de planification économique plus fines des ressources bois-énergie non forestières. 
Négativement, on observe une fatalité face au progrès technique, associée à une certaine appréhension de type Big Brother. Il ressort aussi la crainte qu'une telle précision ne soit source de contraintes accrues dans la mise en œuvre des politiques publiques. En effet, la biodiversité pouvant être caractérisée plus finement, la porte semble ouverte à des mesures conservatoires restrictives à l'échelle des parcelles agricoles. Cette situation est vécue comme une forme d'ingérence dans les pratiques individuelles, notamment de la part de « décideurs » qualifiés d'urbains ou de Toulousains, censés faire peu de cas des préoccupations locales (agriculture et chasse notamment).

\section{Des cartes à dire d'acteurs}

Considérant que les productions cartographiques, tout comme les discussions au cours de l'atelier, traduiraient la coprésence de plusieurs types de représentations de la trame arborée sur le territoire, nous avons proposé aux acteurs d'enrichir leur compréhension de ses multiples variantes par une approche spatialisée, mais également de faire émerger leur perception de la politique de la TVB et de son principal vecteur structurant qu'est la trame arborée. Ce double objectif justifie les constructions scientifiques mais accorde une large place aux collectivités territoriales et aux usagers de l'espace (Alphandéry et al., 2012). L'interprétation qualitative des cartes à dire d'acteurs - représentation spatialisée, légende et discours (Lardon et al., 2001) - rend compte de différentes conceptions de la trame arborée et dégage des perceptions et des enjeux à la croisée des questions de paysage et de biodiversité, ceci dans un jeu de vaet-vient à travers le prisme de la trame arborée.

En première approche, il ressort deux grands types de représentations spatiales de la trame arborée: d'une part, une approche descriptive et factuelle dominée par les ensembles et éléments du paysage, d'autre part, une approche par les processus et les dynamiques paysagères. Ces deux types se démarquent en interne par des différenciations scalaires et de spatialité. Près des deux tiers des participants donnent la priorité aux unités paysagères et aux grands ensembles regroupant les ligneux, le tiers restant se focalise sur les arbres isolés et les réseaux de haies, le reste n'étant considéré que comme des zones plus ou moins forestières. Cependant, près d'un tiers des acteurs représente d'autres éléments sur les cartes, notamment les routes et les espaces bâtis. La trame arborée est ainsi perçue comme un maillage se superposant - en tant qu'espace dual - au réseau de l'habitat et des mobilités. Les représentations spatialisées de la trame arborée apparaissent ainsi comme faisant partie d'une globalité que l'on pourrait qualifier de paysage! Pour la très large majorité apparait également au premier plan la dimension de ressources de l'arbre, qui s'exprime par une valorisation cartographique des vergers et autres arbres isolés considérés comme « utiles ».

De l'analyse plus fine des cartes produites se dégagent finalement quatre types de représentations distinctes. Les trois premiers se distribuent de manière équilibrée entre les acteurs, faisant de ces cartes autant de grilles de lecture de la trame arborée et de ses rapports à la biodiversité et au paysage :

- L'un qu'on pourrait qualifier d'élémentaire se limite à une approche factuelle et descriptive : " Je repère et j'identifie telle grande composante paysagère sur la photographie aérienne. » Cette représentation privilégie les modes d'occupation des sols et oppose de manière un peu 
systématique l'urbain au rural, la forêt aux terres agricoles, sans entrevoir les effets de proximité et de continuité possibles.

Figure 7. Une carte à dire d'acteurs de la trame arborée à Prat-Bonrepeaux (Ariège)

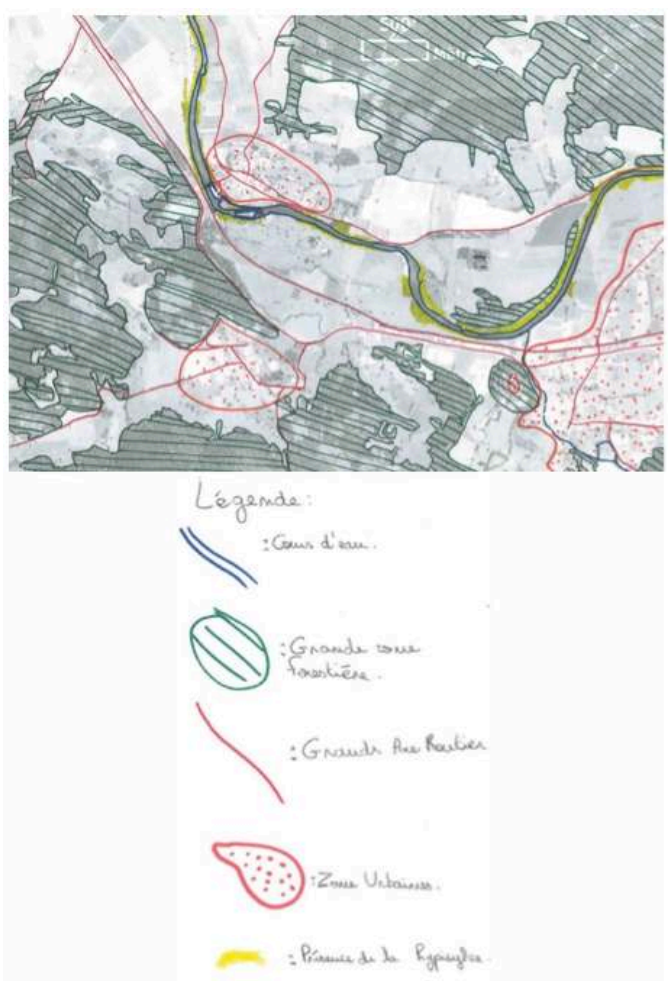

L'auteur a privilégié les contours des grands ensembles paysagers (notamment les masses forestières et les terres agricoles) avec un souci de couvrir tout l'espace, y compris les zones urbanisées.

- Un autre met l'accent sur les dynamiques paysagères, notamment végétales - enfrichement, reforestation -, sans pour autant négliger les transformations du fond de vallée liées à l'urbanisation (petits lotissements) et aux infrastructures (déviation routière de PratBonrepaux). Elle exprime une forte sensibilité aux transformations des paysages agricoles, marquées par une simplification des structures et l'élimination des haies en bord de parcelle, des arbres au milieu des champs... Transparaît aussi en filigrane une certaine nostalgie d'un paysage perdu. 
Figure 8. Une carte à dire d'acteurs de la trame arborée à Prat-Bonrepeaux (Ariège)

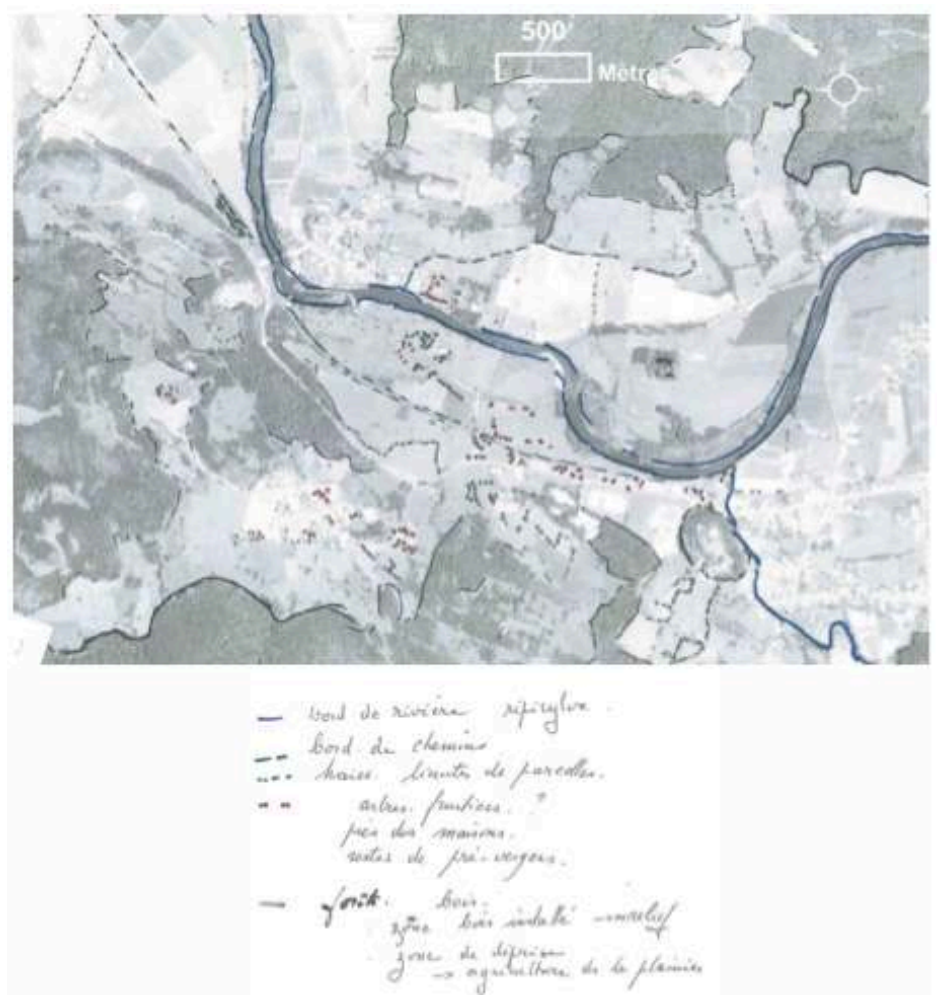

Les processus en cours et historiques servent de fil conducteur à la légende. La carte, a priori pauvre délimite à grands traits les secteurs les plus significatifs de ces dynamiques paysagères.

- Un troisième traduit une lecture fine des composantes de la trame arborée. Celles-ci - haies, arbres isolés, vergers, arbres des maisons et des routes - sont associées à des fonctions productives, esthétiques, de confort et à des usages vernaculaires. Elles sont les marqueurs d'une différenciation paysagère. Elles possèdent fréquemment une forte valeur patrimoniale. 
Figure 9. Une carte à dire d'acteurs de la trame arborée à Prat-Bonrepeaux (Ariège)

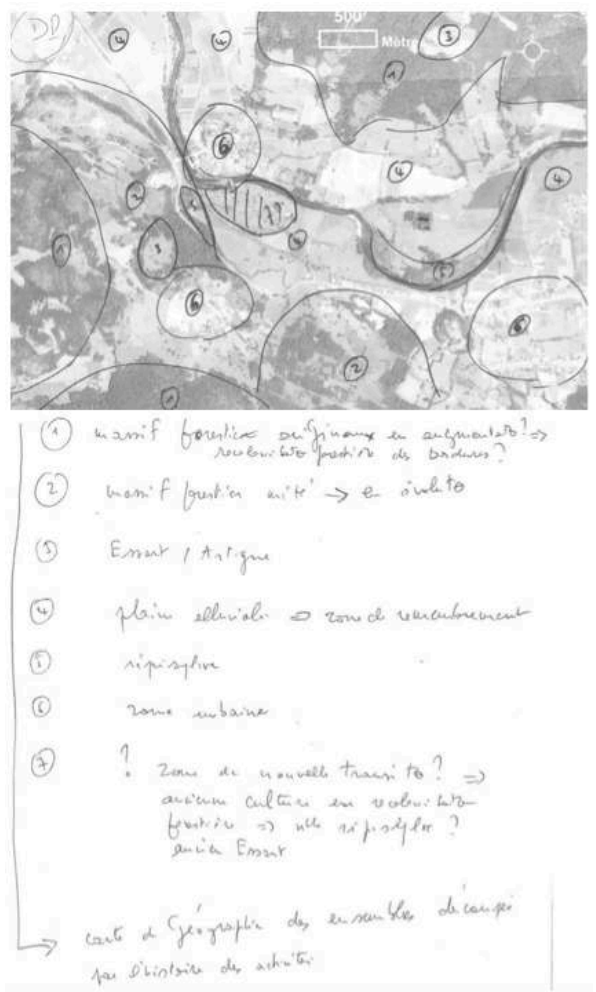

En privilégiant les alignements d'arbres, les arbres isolés, les vergers, associés à d'autres éléments topographiques : rivières, routes, limites de parcelles, bâtis, cette représentation spatiale traduit la finesse des éléments de la trame arborée, supports de biodiversité et de paysage.

- Un dernier, très minoritaire, insiste sur la dimension écologique en faisant explicitement référence au lexique scientifique et institutionnel de la TVB. Réservoirs de biodiversité, corridors écologiques et repérage des discontinuités structurent la spatialisation de la trame arborée. En revanche, ces acteurs font peu de cas des pratiques et des usages locaux. 
Figure 10. Une carte à dire d'acteurs de la trame arborée à Prat-Bonrepeaux (Ariège)

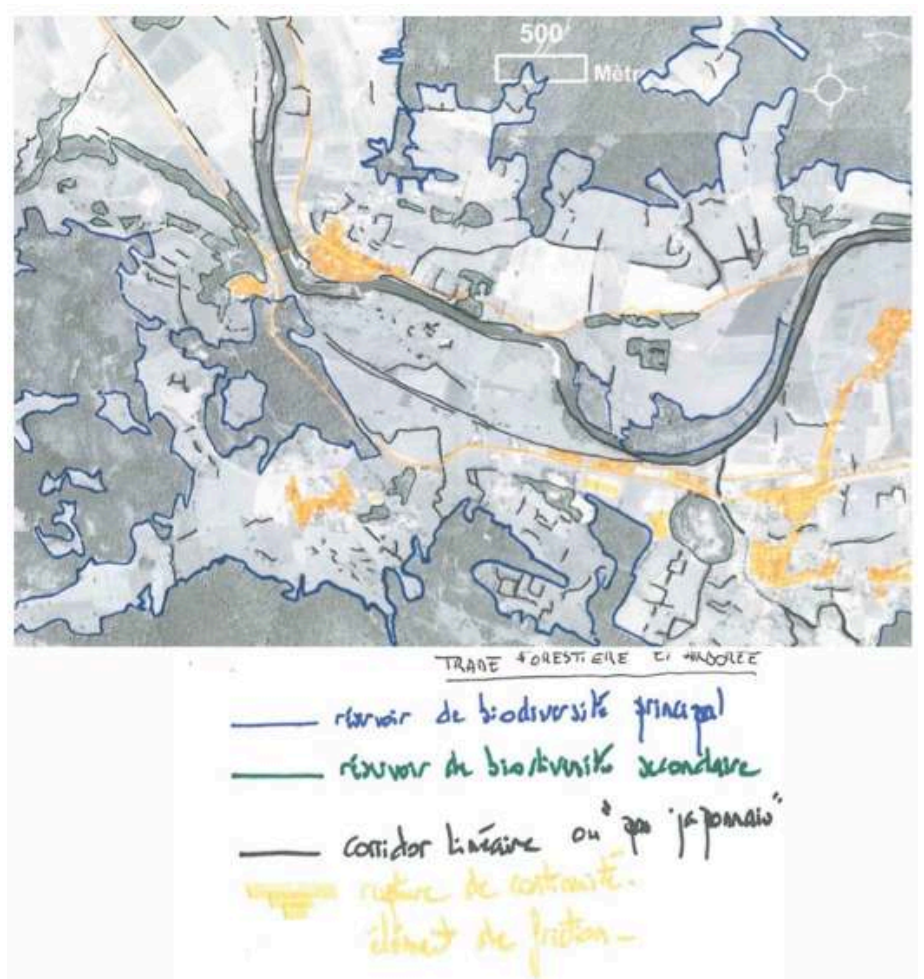

Dans ce focus spatial, un peu à l'écart des grands enjeux de la TVB (SRCE), l'interprétation de la trame arborée au prisme des clés de lecture écologique délaisse tout un pan de la richesse et de la diversité présente.

31 Ces quatre types se répartissent inégalement selon les profils des enquêtés. Ainsi, un agriculteur identifie les grandes entités boisées par rapport aux terres agricoles. Une association promouvant les usages des vergers insiste sur les arbres isolés. Les profils « naturalistes » tentent de montrer des structures en rapport avec la conservation de la biodiversité. Un élu n'oublie pas les processus et enjeux liés à l'urbanisme. Très peu associent, au-delà du repérage et de l'identification spatiale des éléments de la trame arborée, les usages et pratiques alors que les mots employés pour les catégoriser haies, arbres isolés, bocages, alignements - y font référence. Pourtant, le lexique et la terminologie utilisés pour désigner la trame arborée et son explicitation collective font bien ressortir, pour reprendre Guillaume Faburel (2015), différents registres entrecroisés et constitutifs des paysages associés à la trame arborée que sont les pratiques locales, l'imaginaire, le symbolique, le politique et l'écologique :

- celui de patrimoine à travers le repérage, le marquage de l'espace associé à un contenu socioculturel, fonctionnant comme autant de lieux de mémoire ;

- celui de l'objet et de son contenu, c'est-à-dire une structure propre et son fonctionnement avec la diversité de ses composants : forêts, haies...;

- ceux de la production et de la gestion qui met en jeu des pratiques locales, vernaculaires pour certaines d'entre elles, des interventions façonnant les paysages dont les dispositifs d'aménagement et de planification devraient se faire l'écho.

La trame domestique et de proximité liée à l'habitat - qu'il soit agricole ou pavillonnaire - est remarquée pour son intérêt au sein des espaces les plus artificialisés, permettant d'articuler un intérieur et un extérieur. Cette dualité se retrouve dans la 
différenciation qui est faite entre la haie agricole et la haie ou l'arbre des villages et des jardins. Il est tout à fait remarquable que dans une majorité de représentations (à l'exception de la quatrième évoquée ci-dessus), les intérêts et incidences pour la biodiversité sont fort peu présents, quand ils ne sont pas tout simplement absents. La moitié des cartes et des légendes témoignent par ailleurs d'une réelle incompréhension vis-à-vis des expressions " arbre-hors-forêt » et " trame arborée » (rappelons que nous ne les avions pas explicitées préalablement à l'exercice). On mesure ainsi clairement l'importance de l'enjeu d'explicitation et d'information du public, s'agissant des vocabulaires spécialisés.

\section{Éléments de discussion}

Dans l'espace étudié, le dispositif de la trame verte et bleue est aujourd'hui principalement mis en œuvre par des collectivités supracommunales: le parc naturel régional des Pyrénées ariégeoises et les structures porteuses de SRCE/Scot l'intègrent à leurs documents de planification et mettent en place des politiques incitatives à destination des acteurs locaux privés et publics. L'intégration de la TVB au niveau des PLU et PLUI se fait en vertu du principe de subsidiarité qui stipule que les élus locaux doivent largement appliquer les grandes orientations du SRCE et du Scot, afin de laisser une marge de manœuvre entre les échelles de territoire (Allag-Dhuisme, 2012). Il n'en demeure pas moins que ces élus n'ont pas forcément les moyens d'utiliser les outils techniques nécessaires pour alimenter une cartographie haute résolution de la trame arborée dans le PLU.

On observe, d'autre part, une difficulté à comprendre la trame arborée comme concept englobant à la fois l'arbre hors forêt et les massifs forestiers. Il y a là un réel enjeu d'autant que l'approche à partir des paysages fait habituellement mieux sens que la seule cartographie, tant que sa résolution n'est pas fine. C'est à ce niveau que le paysage, quand il est cartographié avec précision, renvoie aussi à des pratiques et des savoirs ordinaires. Les cartes des structures arborées produites par les acteurs locaux établissent clairement le lien entre paysage et biodiversité. Il convient donc de chercher à mieux qualifier le degré de légitimité de ces représentations, entre savoirs individuels et savoirs savants ou experts. Si les évolutions technologiques rendent désormais possible l'établissement d'une carte à haute résolution de la trame arborée, il ne faudra cependant pas occulter la nécessaire étape de l'explication, de la concertation rapprochée et de la coconstruction avec les habitants et usagers des territoires ruraux, et cela en fonction des usages ciblés par les organismes chargés de la mise en place des politiques publiques environnementales.

En effet, la difficulté de prise en compte des trames réside en partie dans un hiatus conceptuel et méthodologique entre les principes qui guident la production d'analyses spatiales depuis l'échelle de la région jusqu'à celle des Scot, et ceux qui devraient fonder la production de connaissances à partir de l'échelon local. Aussi, suggérons-nous de diffuser la connaissance sur la biodiversité au niveau local et d'accorder une légitimité équivalente entre les connaissances scientifiques, les représentations politiques et les savoirs locaux dans un processus de "pluralisation des sources de l'expertise publique »(Blondiaux, 2008). Cette démarche peut être envisagée à l'échelon communal ou intercommunal des PLU, donc avec les acteurs de ces projets. Elle suppose qu'il n'existe pas une seule représentation des trames vertes et bleues sur un 
territoire mais plusieurs, à considérer et à concilier. Cette proposition recoupe les préconisations officielles qui prônent une "adaptation aux réalités locales et aux échelles des documents " (Allag-Dhuisme et al., 2010; Cormier, 2011; Amelot et al., 2013).

Les difficultés de caractérisation des objets territoriaux dues aux divergences des représentations nécessitent donc un exercice de conception innovante de méthodes et outils de production de connaissances, dans un contexte multiacteur. La cartographie, pensée au départ comme objet de connaissance et de communication (médiation), s'avère faisable d'un point de vue technique, mais pas forcément efficiente pour faire progresser la compréhension locale et l'acceptabilité sociale de la politique TVB. Cette situation n'est pas nouvelle (Cormier et al., 2010 ; Cormier, 2014). Mais dans un contexte parfois tendu, où les enjeux territoriaux sont considérés comme relevant de politiques favorisant le développement des activités, et notamment de l'activité agricole dans ces espaces dévitalisés et en voie de patrimonialisation, les confusions sémantiques entre acteurs de terrain et les institutions chargées de sa mise en œuvre attisent les rapports de méfiance. Cependant, l'association des sciences sociales, des sciences spatiales et des sciences écologiques peut favoriser une véritable démarche participative en suscitant l'émergence d'une communauté de pratiques susceptible de construire une vision commune des caractéristiques et des enjeux du territoire et de ses continuités écologiques (Amelot et al., 2013). Force est de constater que les considérations scientifiques qui fondent la légitimité de la démarche TVB (Alphandéry et al., 2012) ne sont pas primordiales pour la plupart des acteurs rencontrés, qui sont plus sensibles sur leur territoire à une nature plus ordinaire, ce que soulignent Catherine Mougenot (2003) ou Sylvie Guillerme et al. (2015), entre autres.

\section{Conclusion}

La cartographie des trames arborées à une précision métrique est un sujet de recherche émergent, en particulier grâce à la plus grande disponibilité des images satellites à haute résolution, mais aussi grâce au développement des techniques par imagerie radar embarquée type Lidar et des drones d'observation. La cartographie de la seule trame arborée à cette précision pourrait exister via la couche OCSGE de l'IGN : les trames arborées devraient être bientôt décrites en douze valeurs attributaires par type de faciès végétal. La cartographie par télédétection à l'aide des images du satellite Pléiades pourrait être programmée chaque année - voire deux fois dans l'année - à un coût raisonnable, dès lors que les images sont mutualisées entre différents partenaires locaux, institutions de recherche scientifique et administrations de l'État. Mais cette perspective reste à étudier du point de vue de sa faisabilité. À cet égard, l'atelier a bien mis en évidence une défiance à l'égard des cartes en résolution métrique, dès lors qu'elles sont susceptibles d'être utilisées en tant qu'outils de planification territoriale.

Cependant, passer d'un réseau fonctionnel conçu par les scientifiques à un projet de territoire partagé par les acteurs locaux reste l'enjeu essentiel pour la réussite de la politique de la TVB. Établir une cartographie précise de la TVB et de l'une de ses principales ossatures, la trame arborée, pourrait être un moyen pour améliorer la cohérence des futures politiques agro-environnementales et diminuer la vulnérabilité des paysages ${ }^{6}$. Les orientations nationales de l'applicabilité de la TVB, et donc de la trame arborée, laissent le choix des méthodes scientifiques et techniques (Allag- 
Dhuisme, 2012), arguant que ce choix résulte d'un compromis entre science et acceptabilité. Toutefois, on ne pourra pas faire l'impasse d'une coconstruction impliquant les acteurs dans leur diversité. Du fait de l'échelle plus fine, cela nécessite d'identifier la trame arborée en intégrant les différents registres et enjeux de biodiversité dont témoigne l'analyse des cartes et des légendes à dire d'acteurs : pratiques locales, imaginaire, symbolique, politique, écologique. Cela suppose notamment des méthodes plus perméables à l'expression locale afin de faciliter une meilleure appropriation de cette nouvelle spatialité fine à l'échelon local pour susciter l'adhésion des différents acteurs: élus, agriculteurs, responsables de territoires protégés et habitants.

Nous tenons ici à remercier le ministère de l'Écologie, du Développement durable et de l'Énergie (MEDDE), qui a financé les recherches du projet Interface dans le cadre du programme PDD2 "Paysage et développement durable».

\section{BIBLIOGRAPHIE}

Allag-Dhuisme F., « La Trame verte et bleue : un outil d'aménagement du territoire pour la préservation de la biodiversité », Colloque « Agriculture et biodiversité. Des pistes pour l'avenir », Val de Drôme, octobre 2012.

Allag-Dhuisme, F. et al., « Guide méthodologique identifiant les enjeux nationaux et transfrontaliers relatifs à la préservation et à la remise en bon état des continuités écologiques et comportant un volet relatif à l'élaboration des schémas régionaux de cohérence écologique. Deuxième document en appui à la mise en œuvre de la trame verte et bleue en France ", proposition issue du comité opérationnel « Trame verte et bleue », MEEDDM, 2010.

Alphandéry, P., Fortier, A., Sourdril, A., « Les données entre normalisation et territoire : la construction de la trame verte et bleue ", Développement durable et territoire, vol. 3, $n^{\circ} 2,2012$, URL : http://developpementdurable.revues.org/9282.

Amelot, X., Bousquet, A., Couderchet, L., Guinard, E., Noucher, M., et al., « Confronter et enrichir les représentations de l'environnement. Vers une mise en interrelation de savoirs hétérogènes sur les continuités écologiques », colloque « Dynamiques environnementales, politiques publiques et pratiques locales : quelles interactions? », Toulouse, juin 2013.

Beringuier, P., Lelli, L., Bertrand, G., « Le réseau paysage Midi-Pyrénées : un dispositif chercheurs-acteurs pour une politique du paysage en région ", Sud-Ouest Européen, $\mathrm{n}^{\circ}$ 38, p. 17-30, 2014.

Blondiaux, L., Le Nouvel Esprit de la démocratie. Actualité de la démocratie participative, Paris, Éditions du Seuil, coll. « La République des idées », 2008, 112 p.

Bonnin, M., Les Corridors écologiques, vers un troisième temps du droit de conservation de la nature?, Paris, L'Harmattan, 2008, 270 p.

Butet, A., Paillat, G., Delettre, Y. « Factors driving small rodent assemblages from field boundaries in agricultural landscapes of western France », Landscape Ecology, vol. 21, 2006, p. 449-461. 
Burel, F., Baudry, J. Écologie du paysage. Concepts, méthodes et applications, Paris, Éditions TEC et DOC, 1999, 359 p.

Clergeau, P., Blanc, N., Trames vertes urbaines. De la recherche scientifique au projet urbain, Paris, Éditions du Moniteur, 2013, 339 p.

Clergeau, P., Une écologie du paysage urbain, Rennes, Éditions Apogée, 2007, 136 p.

Clergeau, P., Désiré, G, « Biodiversité, paysage et aménagement : du corridor à la zone de connexion biologique », Mappemonde, $\mathrm{n}^{\circ}$ 55, 1999-3, p. 19-23.

Cormier, L, « Les Trames vertes : entre discours et matérialités, quelles réalités ? ", thèse de doctorat, université d'Angers, 2011, 368 p.

Cormier, L., « Trames Vertes, vers un nouveau paradigme? », Bulletin de l'association de géographes français, 2014-1, p. 35-51.

Cormier, L., De Lajartre, A. B., Carcaud, N., « La planification des trames vertes, du global au local : réalités et limites ", Cybergeo : European Journal of Geography, juillet 2010, URL : http:// cybergeo.revues.org/23187.

Faburel, G., « La participation citoyenne, chance ou risque pour des projets de paysage ?» colloque de restitution du programme « Paysage et développement durable » (PDD2), Paris, 17 juin 2015.

Guillerme, S., et al., « Dynamiques paysagères et perception de la trame arborée en milieu rural : quels enjeux pour la politique de la trame verte et bleue », dans Luginbühl, Y. (dir.), Biodiversité, Paysage et Cadre de vie, Paris, Victoires Éditions, p. 17-34, 2015.

Guillerme, S., et al., « Interface. Dynamiques paysagères et perceptions des interfaces arborées : quels enjeux pour la mise en place de la trame verte et bleue ? ", rapport final, MEDDE, 2014, URL : http://www.paysage-developpement-durable.fr/IMG/pdf/---guillerme_itf_rapportfinal_v4-2.pdf.

IAU-IDF, « Ecoline, la cartographie des éléments de biodiversité des paysages ruraux », Note rapide, $\mathrm{n}^{\circ} 596$, juillet 2012 .

Lardon, S., Maurel, P., Piveteau, V. (dir.), Représentations spatiales et Développement territorial, Cachan, Hermes Sciences, 2001, 437 p.

Lelli, L., Beringuier, P., Paradis, S., Sirven, B., « Les dispositifs participatifs qui "font dire le

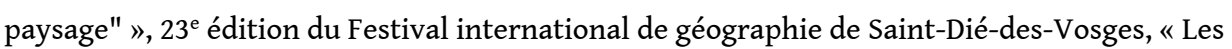
facettes du paysage : nature, culture, économie », 11 au 14 octobre 2012.

Maire, E., et al., « Télédétection de la trame verte arborée en haute résolution par morphologie mathématique ", Revue internationale de géomatique/International Journal of Geomatics and Spatial Analysis, Cachan, Lavoisier, vol. 22, $\mathrm{n}^{\circ}$ 4, 2012, p. 519-538.

Minovez, J.-M., Du papier et des hommes. JOB et les papetiers du Comminges et du Couserans, Arles, Éditions du Rouergue, 2014, 224 p.

Mougenot C., Prendre soin de la nature ordinaire (nouvelle édition), Paris, Éditions de la Maison des sciences de l'homme, 2003, URL: http://books.openedition.org/editionsmsh/1293

Richards, J.A., Xiuping J., Remote Sensing Digital Image Analysis, an introduction, Berlin, SpringerVerlag, 453 p., 2006.

Serra, J., Image analysis and mathematical morphology, vol. 1, London, Academic Press, 1982, 600 p. 
Sheeren, D., Bastin, N., Ouin, A., Ladet, S., Balent, G., Lacombe, J-P, « Discriminating small wooded elements in rural landscape from aerial photography: a hybrid pixel/objectbased analysis approach », International Journal of Remote Sensing, vol. 30, nº 19, 2009, p. 4 979-4 990.

Sheeren, D. et al., « La morphologie mathématique binaire pour l'extraction automatique des bâtiments dans les images THRS ", Revue internationale de géomatique, Cachan, Lavoisier, vol. 17, $n^{\text {os }} 3-4,2007$, p. 333-352.

Tansey, K., Chambers, I., Anstee, A., Denniss, A., Lamb, A., « Oriented classification of very high resolution airborne imagery for the extraction of hedgerows and field margin cover in agricultural areas ", Applied Geography, n² 29, 2009, p. 145-157.

Vanpeene-Bruhier, S. et al., « Efficacité des corridors : qu'en savons-nous vraiment? », Sciences Eaux \& Territoires, $\mathrm{n}^{\circ} 14,2014 / 2$, p. 8-13.

\section{NOTES}

1. http://www.trameverteetbleue.fr/presentation-tvb/qu-est-ce-que-trame-verte-bleue/ definitions-trame-verte-bleue

2. Document disponible en ligne: http://www.midi-pyrenees.developpement-durable.gouv.fr/ IMG/pdf/TVB_minimum_cle6e5f2b.pdf.

3. Les détails techniques sont présentés à l'adresse suivante : http://www.geo-airbusds.com/fr/ 237-pleiades-imagerie-satellite-tres-haute-resolution

4. Document d'objectifs zone Natura 2000 FR 7301822, 2009: http://garonne-midipyrenees.n2000.fr/sites/garonne-midi-pyrenees.n2000.fr/files/documents/page/ DOCOB20Salat20DEF.pdf

5. L'OCSGE définit la couverture et l'usage du sol en France. Depuis 2012, l'IGN est mandaté pour constituer un nouveau référentiel d'occupation du sol à grande échelle et de description du territoire.

6. Il est possible de considérer différentes formes de vulnérabilité comme étant autant de briques de la vulnérabilité paysagère. Le panel est vaste. Nous retiendrons ici notamment en lien avec la TVB : les risques liés aux crues et aux inondations, les extensions urbaines mal maîtrisées, et l'érosion de la biodiversité due aux changements paysagers et aux modifications des pratiques agricoles et pastorales.

\section{RÉSUMÉS}

La trame verte et bleue (TVB) est l'une des mesures phares issues du Grenelle II de l'environnement. Elle s'impose, à travers les schémas régionaux de cohérence écologique (SRCE), aux documents d'aménagement et d'urbanisme. La trame arborée, en tant que structure paysagère, est une composante essentielle de la TVB. Elle reste cependant fréquemment mal identifiée aux diverses échelles spatiales, particulièrement en milieu rural. Les cartographies et préconisations de grands corridors écologiques à moyenne échelle (1/50 000 dans le meilleur des cas) ont certes leur utilité, mais ne reflètent pas réellement les enjeux locaux de biodiversité. Nous montrerons, à travers un exemple dans le piémont pyrénéen ariégeois, qu'il existe des 
méthodes pour réaliser des cartographies fines des trames arborées, en utilisant les images des satellites Pléiades. Ces outils de connaissance ont été testés lors d'un atelier participatif qui a mis en évidence une grande diversité de représentations de la trame arborée parmi les acteurs locaux.

The Green and Blue Infrastructure is one of the flagship initiatives which resulted from the second French environmental round table. It is implemented via regional plans for environmental coherence, which have an impact on regional and urban planning documents. The green corridor, as a structural element of the landscape is an essential component of the Green and Blue Infrastructure. However, it is frequently poorly identified at the different spatial levels, especially in rural areas. The maps and planning documents of major ecological corridors on a medium scale (1/50 000 in the best cases) are useful, however they do not realistically reflect local biodiversity challenges. We shall demonstrate through an example in the Pyrenean Piedmont Ariège region, that there are viable methods for making detailed maps of green corridors by using images from the Pléaides satellites. These knowledge tools have been tested in an interactive workshop which revealed a great variety of representations of the green corridor among local stakeholders.

\section{INDEX}

Keywords : green corridor, stakeholder maps, representations, high definition remote sensing maps, landscape

Mots-clés : trame arborée, cartes à dire d'acteurs, représentations, cartes haute résolution par télédétection, paysage

\section{AUTEURS}

\section{ÉRIC MAIRE}

Éric Maire est ingénieur de recherche CNRS en traitement, analyse et représentation de l'information spatiale, laboratoire GEODE - UMR 5602 CNRS (Géographie de l'environnement). eric.maire[at]univ-tlse2[dot]fr

\section{PHILIPPE BÉRINGUIER}

Philippe Beringuier est maître de conférences en géographie et aménagement, paysages en transition, à l'université Toulouse - Jean Jaurès, laboratoire GEODE - UMR 5602 CNRS

(Géographie de l'environnement).

philippe.beringuier[at]univ-tlse2[dot]fr

\section{GÉRARD BRIANE}

Gérard Briane est géographe (biodiversité des milieux naturels et agricoles), il est maître de conférence à l'université Toulouse - Jean Jaurès, laboratoire GEODE - UMR 5602 CNRS

(Géographie de l'environnement).

briane[at]univ-tlse2[dot]fr

\section{BERTRAND DESAILLY}

Bertrand Dessailly est géographe (environnement et paysage urbain, géohistoire des risques) et maître de conférence à l'université Toulouse - Jean Jaurès. Laboratoire GEODE - UMR 5602 CNRS 
(Géographie de l'environnement).

desailly[at]univ-tlse2[dot]fr

\section{SYLVIE GUILLERME}

Sylvie Guillerme est géographe, spécialisée en agroforesterie et agrobiodiversité, et chargée de recherche au CNRS, laboratoire GEODE - UMR 5602 CNRS (Géographie de l'environnement). sylvie.guillerme[at]univ-tlse2[dot]fr 